\title{
Research on adaptive event triggering schemes based on network control systems (NCSs)
}

\author{
Zhang Li \\ Department of Mathematics \& Physics . Hohai University, Changzhou, China \\ Email: zhangl@hhuc.edu.cn
}

Keywords : Networked Control System (NCS), Adaptive event-triggering scheme

Abstract: In this paper, a review of some of the existing event-triggering schemes and system, in which they are implemented is discussed. A summary of the main ideas of the event-triggering schemes is given. At the end of paper, the motivation for this research is given in detail.

\section{Introduction}

The advancement of technology increased the application based on control systems compared with uncontrolled systems. Control systems often guarantee the stability and lead to improved performance. They can be implemented in simple systems such as washing machine, robust system and satellite systems. Traditionally all control systems had their components (sensors, actuators, and controller) connected directly to each other, but with many innovations in the field new control systems called networked control systems have been introduced. A NCS is a control system in which the components are connected by a digital communication network[1]. Due to the advantages associated with NCSs, the interest in this field has increased exponentially in the past few years. The advantages include reduction in implementation and maintenance cost, complexity, weight and power requirements of system [2-8]. However, the introduction of a digital communication network in a control system also creates new challenges in the modeling, design and analysis of the system. These challenges can significantly reduce the performance of the control system as well as destabilize the whole system. The challenges include packet dropout, quantization, network and computational induced time delay [9-15].

\section{A static event-triggering scheme}

Peng, $C$ in [16] designed a static event-triggering scheme and implemented it in an event generator located between the sensor and the controller. Peng made several assumptions, which enabled him to implement the event-triggering scheme. The stated assumptions are

1.The state of the system is periodically sampled with a constant sampling $h>0$.

2.The event-triggering scheme determines which of the sampled data is transmitted. The transmission instants are denoted by $\left\{t_{0} h, t_{1} h, t_{2} h, \ldots.\right\}$, where $t_{0} h=0$.

3.The zero-order hold $(\mathrm{ZOH})$ generates the control input of the system, with a holding interval of $t \in \Omega=\left[t_{k} h+\eta_{1}, t_{k+1} h+\eta_{1}\right)$ where $\eta_{1}$ is the constant network-induced delay.

\section{Model}

The system which is considered in [16] is given as 


$$
A(t)=A x(t)+B u(t)+B_{1} \omega(t)
$$

where $x(t) \in^{\circ n}$ is state vector, $x_{0} \in^{\circ n}$ is the initial condition, $u(t) \in^{\circ m}$ is control input vector and $\omega(t) \in L_{2}[0, \infty)$ is the disturbance. The static feedback controller is given as

$$
u(t)=K x\left(t_{k} h\right)
$$

where $K$ is the controller gain and $x\left(t_{k} h\right)$ is the last transmitted data. To help implement the event-triggering scheme in the system, the holding interval of ZOH (Zero Order Hold), was divided into subintervals

$$
\Omega=\cup \Omega_{l} \text { where } \Omega_{l}=\left[i_{k} h+\eta_{1}, i_{k} h+h+\eta_{1}\right)
$$

where $l=0,1, \ldots, t_{k+1}-t_{k}-1, \quad i_{k}=t_{k}+l$. Then by defining $\eta(t)=t-i_{k} h, \quad t \in \Omega_{l}$ as a piecewise function which satisfies

$$
\eta(t)=1, \eta_{1} \leq \eta(t) \leq h+\eta_{1} @ \eta_{2}
$$

Then, the controller law (2)becomes

$$
u(t)=K\left(x(t-\eta(t))-e\left(j_{k} h\right)\right), \quad t \in \Omega_{l}
$$

where $e\left(j_{k} h\right) @ x\left(j_{k} h\right)-x\left(t_{k} h\right)$ is the threshold error between the current sampled data and the latest transmission. Combining (1) and (5) will result in the following closed loop system

$$
A(t)=A x(t)+B K x(t-\eta(t))-B K e\left(j_{k} h\right)+B_{1} \omega(t) \quad t \in \Omega_{l}
$$

\section{Event-triggering condition}

In [16] in the event generator computes the sampled-error between the current sampled data and the last transmitted data. A specified threshold will determine whether sampled data is transmitted or not. The sampled data is only transmitted when the threshold is violated. The next transmitted instant of the event-triggering scheme is given as

$$
t_{k+1} h=t_{k} h+\min _{i \geq 0}\left\{(i+1) h \mid e^{T}\left(j_{k} h\right) \Phi e\left(j_{k} h\right) \geq \delta x^{T}\left(t_{k} h\right) \Phi x\left(t_{k} h\right)\right\}
$$

where $i \in \bullet, j_{k}=t_{k}+i+1, \delta>0$ is the threshold and $\Phi>0$ is the weighting matrix.

\section{Interpretation}

The event-triggering scheme (7) significantly reduces the amounted of data packets which are transmitted between components in a NCSs. This event-triggering scheme surpasses the main objective of event triggered control objective, which is to reduce the amount of data transmitted as well as maintaining satisfactory performance of the system. However, comparing the event-triggering mechanism presented in [16] with periodic communication scheme, the 
performance of the closed-loop system has been degraded because less information of the controlled plant is used for feedback. The event-triggering scheme also ensure that there is no Zeno behavior[17], since the lower bound of the inter event time $t_{k+1} h-t_{k} h$ is the sampling period $h>0$.

\section{A adaptive event-triggering schemes}

In this subsection, two adaptive event-triggering schemes employed in NCSs are investigated. The first one adaptively adjust the triggering parameter with respect to the dynamic error of the system. The second, one considers the variations in the limited resources of the communication network and fault occurrence probability when designing the event-triggering scheme.

\section{An adaptive event-triggering scheme for an error dynamic system}

In [18] an event-triggered control method for a networked control system with randomly occurring nonlinearities and uncertainties under network-induced delay is considered. The authors in [18] made the following assumptions

The sensors are clock driven, while the controller and actuator are event driven;

The computational delay and the network-induced delays are combined together as $\eta_{k} \in\left[\eta_{m}, \eta_{M}\right]$ where $\eta_{m}$ and $\eta_{M}$ are the lower and upper bounds of $\eta_{k}$ respectively;

The event-triggering scheme determines which of the sampled data is transmitted. The transmission instants are denoted by $\left\{t_{0} h, t_{1} h, t_{2} h, \ldots.\right\}$, where $t_{0} h=0$.

\section{Model}

The system considered in [18] is described by

$$
\delta(t)=\left(A_{0}+\alpha(t) \Delta A\right) x(t)+\left(B_{0}+\beta(t) \Delta B(t)\right) u(t)+\gamma(t) h(t, x(t))
$$

where $x(t) \in^{\circ n}$ is the state vector, $x_{0} \in^{\circ n}$ is the initial condition, $u(t) \in^{\circ m}$ is control input vector. $A_{0}$ and $B_{0}$ are constant matrices with appropriate dimensions. $\Delta A(t)$ and $\Delta B(t)$ represent the parameter uncertainties satisfying

$$
[\Delta A(t) \quad \Delta B(t)]=G F(t)\left[\begin{array}{ll}
E_{a} & E_{b}
\end{array}\right]
$$

where $G, E_{a}$ and $E_{b}$ are constant matrices with appropriate dimension and $F(t)$ is an unknown time-varying matrix satisfying $\left\|F^{T}(t) F(t)\right\| \leq I . h(t, x(t))$ is a piecewise-continuous nonlinear function which satisfies

$$
h^{T}(t, x(t)) h(t, x(t)) \leq \kappa^{2} x^{T}(t) H^{T} H x(t)
$$

where $\kappa>0$ is the bounding parameter on the uncertain function $h(t, x(t))$ and $H$ is a constant matrix. The networked state feedback controller is designed as: 


$$
u(t)=K x\left(t_{k} h\right) \quad t \in\left(t_{k} h+\eta_{k}, t_{k+1} h+\eta_{k+1}\right]
$$

(11)

where $K$ is the controller gain to be determined and $x\left(t_{k} h\right)$ is the latest transmitted sampled data. By letting $l_{k}=\min \left\{n \mid t_{k} h+n h+\eta_{k} \geq t_{k+1}+\eta_{k+1}\right\}$, Then the $\left(t_{k} h+\eta_{k}, t_{k+1} h+\eta_{k+1}\right]$ can be expressed as

$$
\left[t_{k} h+\eta_{k}, t_{k+1} h+\eta_{k+1}\right)=\bigcup_{n=0}^{l_{k}-1} \Omega_{n}
$$

where

$$
\begin{aligned}
\Omega_{n} & =\left[t_{k} h+n h+\eta_{k}, t_{k+1} h+n h+\eta_{k+1}\right), n=0, \ldots ., l_{k}-2 \\
\Omega_{l_{k}-1} & =\left[t_{k} h+l_{k} h+\eta_{k}, t_{k+1} h+\eta_{k+1}\right)
\end{aligned}
$$

Then by defining $\tau(t)=t-i_{k} h$, where $i_{k} h=t_{k} h+n h, t \in \Omega_{n}$ as a piecewise function which satisfies

$$
\eta_{m} \leq \eta_{k} \leq \tau(t) \leq h+\max \left\{\eta_{k}, \eta_{k+1}\right\} \leq h+\eta_{M}, t \in \Omega_{n}
$$

Then, the controller law (11) becomes

$$
u(t)=K\left(x(t-\eta(t))-e\left(i_{k} h\right)\right), \quad t \in \Omega_{n}
$$

where $e\left(i_{k} h\right) @ x\left(i_{k} h\right)-x\left(t_{k} h\right)$ is the threshold error between the current sampled data and the latest transmission. Combining (8) and (14) will result in the following closed loop system

$$
A(t)=A x(t)+B K x(t-\tau(t))-B K e\left(i_{k} h\right)+\gamma(t) h(t, x(t)) \quad t \in \Omega_{n}
$$

where $A=A_{0}+\alpha(t) \Delta A(t), \quad B=B_{0}+\beta(t) \Delta B(t)$.

\section{Event-triggering condition}

In [18] an adaptive event-triggering scheme was proposed, which determines whether the current sampled data should be transmitted, to effectively utilize the limited resources in a communication network. The next transmission instant $t_{k+1} h$ can be determined by

$$
t_{k+1} h=t_{k} h+\min \left\{n h \mid e^{T}\left(i_{k} h\right) \Phi e\left(i_{k} h\right)>\sigma\left(i_{k} h\right) x^{T}\left(t_{k} h\right) \Phi x\left(t_{k} h\right)\right\}
$$

where $n \in \bullet, i_{k}=t_{k} h+n h, h$ is the sampling period of the sensor, and $\Phi>0$ is the weighting matrix. The triggering parameter $\sigma\left(i_{k} h\right)$ is assumed to take values on either $\sigma_{1}$ or $\sigma_{2}$ with 


$$
\sigma\left(i_{k} h+h\right)= \begin{cases}\sigma_{1} & \text { if } e^{T}\left(i_{k} h\right) e\left(i_{k} h\right) \geq \Delta \\ \sigma_{2} & \text { if } e^{T}\left(i_{k} h\right) e\left(i_{k} h\right)<\Delta\end{cases}
$$

where $0 \leq \sigma_{1}<\sigma_{2} \leq 1$ are known constants and $\Delta$ is allowed threshold error.

\section{Interpretation}

The adaptive event-triggering scheme proposed in [18] saves network resources by switching between the two event trigger parameter. That is to say if the error between the current sampled data and the latest transmitted data is large, then more data is transmitted to the controller and if there is a small threshold error then less data is transmitted to the controller. This adaptive event-triggering scheme provides a balance between the system performance and network resources. This event-triggering mechanism surpassed the event-triggering scheme presented in [16].

\section{Conclusions and Motivation}

From the surveyed work shows that adaptive event-triggering schemes outperforms the static event-triggering schemes. From the aforementioned sections, the adaptive event-triggering scheme provides a balance between the system performance and the network resources effectively. So designing an adaptive event-triggering scheme that takes into account the variations in a network load is a necessity since such a scenario occurs in practical communication network. This will be first motivation of this thesis. Up to now, few works have been presented in literature on the stabilization and control for NCSs with simultaneous consideration of network-induced time-varying delay and an adaptive event-triggered communication scheme. This will be the second motivation of this thesis. In this thesis, the state feedback controller design methods for the event-triggered NCSs with time varying delays are presented

\section{References}

[1]. Zhang, W.,Branicky, M.S., and Phillips, S.M., Stability of networked control systems. IEEE Control Systems, 2001, 21(1): pp. 84-99.

[2].Bushnell, L.G., Networks and control. IEEE Control Systems Magazine, 2001. 21(1): pp. 22-23.

[3].Farnam, A. and Esfanjani, R.M. ,Improved stabilization method for networked control systems with variable

transmission delays and packet dropout. ISA transactions, 2014. 53(6): pp. 1746-1753.

[4].Fridman, E., A refined input delay approach to sampled-data control. Automatica, 2010. 46(2): pp. 421-427.

[5].Gao, H., Wu, J., and Shi, P., Robust sampled-data $\mathrm{H} \infty$ control with stochastic sampling. Automatica, 2009, 45(7): pp. 1729-1736.

[6].Lu, R., et al., Networked control with state reset and quantized measurements: observer-based case. IEEE Transactions on Industrial Electronics, 2013, 60(11): pp. 5206-5213.

[7].Rahmani, B., Markazi, A.H., and Seyfi, B., A new method for control of networked systems with an experimental verification. ISA transactions, 2015, 56: pp. 299-307.

[8].Gao, H., Meng, X., and Chen, T.,Stabilization of networked control systems with a new delay characterization. IEEE Transactions on Automatic Control, 2008, 53(9): pp. 2142-2148.

[9].Walsh, G.C., Ye, H., and Bushnell, L.G., Stability analysis of networked control systems. IEEE transactions on control systems technology, 2002, 10(3): pp. 438-446. 
[10].Ugrinovskii, V. and Fridman, E. , A Round-Robin type protocol for distributed estimation with Hœ consensus. Systems \& Control Letters, 2014, 69: pp. 103-110.

[11].Quevedo, D.E., Silva, E.I., and Goodwin. G.C., Packetized predictive control over erasure channels. in 2007 American Control Conference. 2007, IEEE.

[12].Zhang, L., Gao, H., and Kaynak, O., Network-induced constraints in networked control systems - a survey. IEEE transactions on industrial informatics, 2013, 9(1): pp. 403-416.

[13].Gao, H., Chen, T. , and Lam, J., A new delay system approach to network-based control. Automatica, 2008, 44(1): pp. 39-52.

[14].Yue, D., Han, Q.-L., and Peng. C., State feedback controller design of networked control systems. in Control Applications, 2004, Proceedings of the 2004 IEEE International Conference on. 2004. IEEE.

[15].Peng, C., et al., On hold or drop out-of-order packets in networked control systems. Information Sciences, 2014. 268: pp. 436-446.

[16].Peng, C. and Q.-L. Han, A novel event-triggered transmission scheme and control co-design for sampled-data control systems. IEEE Transactions on Automatic Control, 2013, 58(10): pp. 2620-2626.

[17].Donkers, M. and Heemels, W. , Output-based event-triggered control with guaranteed-gain and improved and decentralized event-triggering. IEEE Transactions on Automatic Control, 2012. 57(6): p. 1362-1376.27.

[18]. Zhang, H., et al., Adaptive model-based event-triggered control of networked control system with external disturbance. IET Control Theory \& Applications, 2016. 10(15): p. 1956-1962. 